\title{
Improved Comb Filter Based Approach for Effective Prediction of Protein Coding Regions in DNA Sequences
}

\author{
Jayakishan Meher ${ }^{1}$, Pramod K. Meher ${ }^{2}$, Gananath Dash ${ }^{3}$ \\ ${ }^{1}$ Department of Electronics \& Telecommunication Engineering, SITE, Orissa, India; ${ }^{2}$ Department of Embedded Systems, Institute for \\ Infocomm Research, Singapore City, Singapore; ${ }^{3}$ Department of Physics, Sambalpur University, Orissa, India. \\ Email: jk_meher@yahoo.co.in, pkmeher@i2r.astar.edu.sg, gndash@ieee.org
}

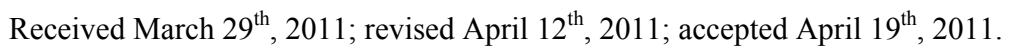

\begin{abstract}
The prediction of protein coding regions in DNA sequences is an important problem in computational biology. It is observed that nucleotides in the protein coding regions or exons of a DNA sequence show period-3 property. Hence identification of the period-3 regions helps in predicting the gene locations within the billions long DNA sequence of eukaryotic cells. The period-3 property exhibited in exons of eukaryotic gene sequences enables signal processing based time-domain and frequency domain methods to predict these regions efficiently. Several approaches based on signal processing tools have, therefore, been applied to this problem, to predict these regions effectively. This paper describes novel and efficient comb filter-based techniques for the prediction of protein coding region based on the period-3 behavior of codon sequences. The proposed method is then validated on Burset/Guigo1996, HMR195 and KEGG standard datasets using various prediction measures. It is shown that cascaded differentiator comb (CDC) filter can be used for prediction of protein coding region with better prediction efficiency, and involves less computational complexity compared with the other signal processing techniques based on period-3 property.
\end{abstract}

Keywords: Cascaded Differentiator Comb (CDC), Generalized Comb Filter (GCF), Indicator Sequence, Period-3, Signal Processing

\section{Introduction}

The genomic information is found to be embodied in the strands of DNA as sequences of tri-nucleotide called codons. A nucleotide is said to be of coding type if it belongs to an exon or of non-coding type if it belongs to an intron or intergenic space. In eukaryotes, the exons are found to be separated by introns, where as in prokaryotes they are placed continuously without any introns in between. Computational gene prediction is based on mainly by two classes of methods such as sequence similarity searches and gene structure and signal-based searches [1]. Exon detection must rely on the content sensors, which refer to the patterns of codon usage that are unique to a species, and allow coding sequences to be distinguished from the surrounding non-coding sequences by statistical detection algorithms. Many algorithms are applied for modeling gene structure, such as dynamic programming, linear discriminant analysis, Linguistic methods, Hidden
Markov model and neural network. Based on these models, a great number of gene prediction programs have been developed [1]. Recently signal processing approach has played a major role in gene prediction using period-3 property.

The protein coding regions of DNA sequences exhibit a period-3 behavior which results specifically from the existence of the codon sequences. Period-3 property is the short range periodicity and is one of among many types of periodicity in DNA sequence. Identification of period-3 regions therefore helps in predicting the gene locations; and allows the prediction of specific exons within the genes of eukaryotic cells [1-3]. In order to predict the location of protein coding region, a sliding data frame (sliding window) with a small step size is employed. This technique has been widely used to identify the coding region which can predict whether a given sequence of frame, limited to a specific length $N$ (called 
as window), belongs to a coding region or not. This is done by moving the sequence frame in which the nucleotides of length $N$ of the window are rated at specific position. The existence of three-base periodicity exhibited by the genomic sequence as a sharp peak at frequency $f=$ $1 / 3$ in the power spectrum in the protein coding regions helps in the prediction of exons. The genomic signal processing involves conversion of DNA character-string into numerical sequence called as the indicator sequence. In addition to the Voss representation [4] which involves binary representation, various DNA numerical signal representations have been adopted using complex numbers [5], quaternion [6], EIIP [7,8], Gailos field assignment [9], frequency of nucleotide occurrence [10], z-curve [11,12], paired numeric [13] to make indicator sequence in DSP methods to improve the sensitivity and selectivity.

The existing DSP techniques for the identification of protein coding regions of DNA sequences based on the period-3 behavior differ in terms of computational complexity and accuracy of prediction. Discrete Fourier transform is used to detect period-3 property in DNA sequences [14-17]. The DFT of length $N$ for input indicator sequence $x_{B}(n)$ is defined by

$$
X_{B}(k)=\sum_{n=0}^{N-1} x_{B}(n) \cdot \mathrm{e}^{-j 2 \pi k n / N}, 0 \leq k \leq N-1
$$

for $B=A, T, C$ and $G$. The absolute value of power of DFT coefficients is given by

$$
S(k)=\sum_{k=0}^{N-1}\left|X_{B}(k)\right|^{2}
$$

The plot of $S(k)$ against $k$, results in peak at $k=N / 3$ due to the period-3 property, that indicates the presence of coding regions.

The digital filtering techniques such as the antinotch filter and multistage filter have been used to identify period-3 property in DNA sequences $[18,19]$. In digital filtering method for indicator sequence $X_{B}(n)$, corresponding filter output $Y_{B}(n)$ is computed where $B=A, T$, $C$ and $G$. The sum of the square of filter outputs is expressed as

$$
Y(n)=\sum_{n=0}^{N-1}\left|Y_{B}(n)\right|^{2}
$$

A plot of $Y(n)$ has been used to extract the period-3 region of the DNA sequence effectively. Gene prediction in eukaryotes based on the DFT by spectral rotation measure is presented by Koltar and Lavner [20]. Short time Fourier transform (STFT) has also been used as a predictor for coding region for improving computational load. Entropy based methods with this predictor is used to increase its efficacy to identify the homogeneous regions. It has been used to identify the borders between coding and noncoding regions in DNA sequence based on the entropy measures with a 12-symbol alphabet [21]. The 3-periodicity is explained in more detail by Tuqan and Rushdi [22] as related to the codon bias using two stage digital filter and multirate DSP model. Modified Gabor-Wavelet transform is used by Jesus et al. [23] for the identification of protein coding regions having advantage of being independent of the window length. The spectrum for DNA sequences is discussed based on an entropy minimization criterion by Galleani and Garello [24]. Criteria to select the numerical values to represent genomic sequences are discussed by Akhtar et al. [25] and in addition a technique for recognition of acceptor splice sites is discussed.

The exon identification task carried out by existing methods has its own limitations as it is observed that period-3 property is not exhibited in some coding regions. Sometimes they do exhibit, but the signal is rather weak and difficult to differentiate from noise. Again false exons are identified and very short exons are missed which are traditional problems in gene prediction history. Due to this gene prediction problem still remains a challenging task in terms of better accuracy, sensitivity and selectivity using existing tools. In such situations shortcomings of the previous approaches motivate to develop new approaches to have improved accuracy and less computational complexity.

In this paper, two new signal processing tools namely the generalized comb filter (GCF), and cascaded differentiator comb (CDC) filter, are presented that effectively use the period-3 property in a genomic sequence for the prediction of protein coding regions. The GCF-based method has lower computational complexity and provides better identification of coding regions over existing DSP methods. The CDC-based approach is an extension of GCF that exploits period-3 behavior more effectively and reduces the computational complexities further. In order to validate the results of the proposed predictor, prediction measures such as discriminating factor, sensitivity, specificity, miss rate and wrong rate are evaluated with HMR195, Burset and Guigo and KEGG standard data sets.

The rest of the paper is organized as follows. Section-2 presents the proposed computationally efficient comb filter-based approach with GCF and CDC filter for the identification of protein coding regions. Section-3 presents the comparison of performances in terms of prediction measures and computational complexities of various signal processing methods and Section-4 presents the conclusions of this paper. 


\section{Proposed Comb Filter Based Approach}

\subsection{Design of a Comb Filter for Identifying Protein Coding Regions}

A comb filter has a frequency response that is periodic function of $\omega$ with a period $2 \pi / L$, where $L$ is a positive integer. Amplitude response of comb filter is comprised of a series of regularly spaced spikes of interleaved passbands and stopbands which looks like a hair comb. A comb filter can also be viewed as a notch filter in which the notches or the nulls occur periodically across the frequency band [26,27]. A comb filter can, thus, be generated from a filter $G(z)$ with single passband and/or a single stopband by replacing each delay in its realization with $L$ delays, resulting in a structure with a transfer function given by

$$
H(z)=G\left(z^{L}\right)
$$

such that if the amplitude response $\left|H\left(e^{j \omega}\right)\right|$ exhibits a peak at $\omega_{p}=\pi / 2$, then the amplitude response of $\left|G\left(e^{j \omega}\right)\right|$ exhibits $L$ peaks at $\pi k / 2 L$, for $1 \leq k \leq L$.

A simplest form of comb filter can be realized by adding a delayed version of a signal to itself or the current filter output to cause constructive and destructive interferences. Comb filters can accordingly be realized in two different forms, e.g., feed-forward form and feedback form. The feed-forward form implements a finite impulse response (FIR) filter while the feedback form implements an infinite impulse response (IIR) filter. The difference equation of a comb filter can be written in a general form:

$$
y(n)=\left[b_{0} x(n)-b_{1} x\left(n-n_{1}\right)\right]+a y\left(n-n_{2}\right)
$$

where $b_{1}$ and $a$, respectively, denote the feed-forward and feedback gain coefficients, $n_{1}$ and $n_{2}$ are fixed delays, $x(n)$ denotes the $n$th sample of the input signal, $y(n)$ is the output at time instant $n$. Equation (5) refers to an FIR filter when the feedback coefficient $a=0$. Taking the $z$-transform of both sides of (5) we can get the transfer function of comb filter to be

$$
Y(z)=b_{0} X(z)-b_{1} z^{-n_{1}} X(z)+a z^{-n_{2}} Y(z)
$$

where $X(z)$ and $Y(z)$ are the z-transform of the input and the output signals, respectively. The transfer function of a general comb filter can thus be obtained to be

$$
H(z)=Y(z) / X(z)=b_{0} \cdot z^{\left(n_{2}-n_{1}\right)}\left[\frac{z^{n_{1}}-b}{z^{n_{2}}-a}\right]
$$

where $b=b_{1} / b_{0}$. The transfer function of feed-forward and feedback type comb filter as shown in (8) and (9) is derived by substituting $b=0$ and $a=0$ in (7), respectively where $n_{1}=n_{2}=L$.

$$
\begin{aligned}
& H_{f}(z)=b_{0}\left(\frac{z^{L}-b}{z^{L}}\right) \\
& H_{b}(z)=b_{0}\left(\frac{z^{L}}{z^{L}-a}\right)
\end{aligned}
$$

From (8) we can find that the numerator equals to zero whenever $z^{L}=b$, which is satisfied at $L$ equally spaced points around a circle in the complex $z$-plane, which form the zeros of the transfer function. Since the denominator is zero at $z^{L}=0, L$ poles would exist at $z=0$. Similarly, from (9) we can find that the numerator equals to zero at $z^{L}=0$, which gives $L$ zeros at $z=0$. The denominator of (9) equals to zero when $z^{L}=a$, which results in $L$ equally spaced poles of the transfer function around a circle in the complex $z$-plane. The signal flow-graph for a comb filter defined by the difference equation of (5) and the pole-zero plot of feed forward and feedback form comb filter are shown in Figure 1. and Figure 2 respectively. From (8) and (9), we can find the amplitude responses of the feedforward and the feedback comb filters, respectively as

$$
\begin{aligned}
& \left|H_{f}(\omega)\right|=b_{0} \sqrt{\left(1+b^{2}\right)-2 b \cos (\omega L)} \\
& \left|H_{b}(\omega)\right|=b_{0} / \sqrt{\left(1+a^{2}\right)-2 a \cos (\omega L)}
\end{aligned}
$$

It can be observed from (10) and (11), that the amplitude

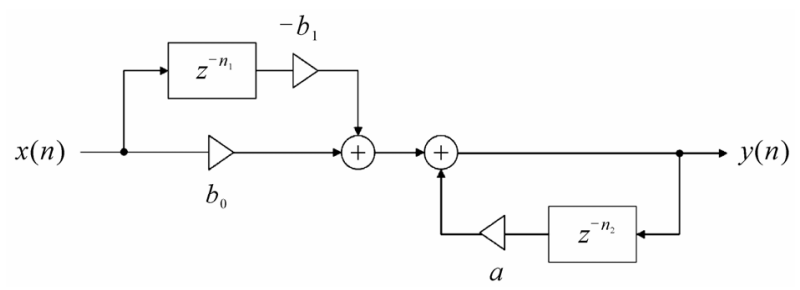

Figure 1. The signal flow graph for a comb filter defined by the difference equation of (5).

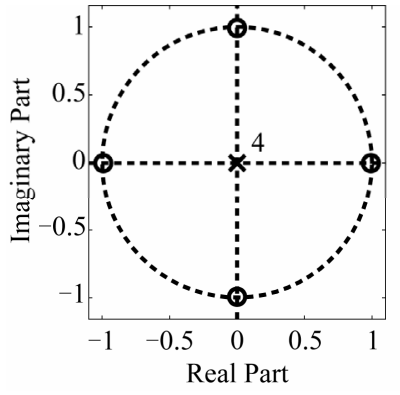

(a)

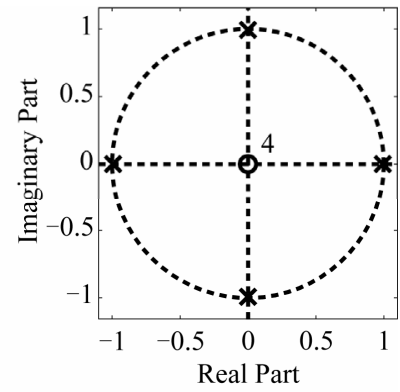

(b)
Figure 2. The pole-zero plots of feedforward and feedback comb filters defined by the transfer functions of (8) and (9) for $L=4$. (a) For feedforward comb filter. (b) For feedback comb filter. 
response of both feedforward and feedback filters vary periodically with frequency $\omega$ with a period of $2 \pi / L$. The behaviour of amplitude responses of both these classes of comb filters are shown in Figure 3 for different values of coefficients $a$ and $b$. As shown in Figure 3(a) the magnitude response of feedforward filter periodically drops to a local minimum $b_{0}(1-b)$ and goes up to a local maximum $b_{0}(1+b)$ resulting in a series of interleaved notches and peaks symmetrically across the line $|H(\omega)|=$ $b_{0}$. For $b=1$ the local minimum goes to zero and becomes closer to $b_{0}$ for decreasing values of $b$. The magnitude response of feedback comb filter is shown in Figure 3(b). In this case the response value periodically drops to a local minimum $b_{0} /(1+a)$ and goes up to a local maximum $b_{0} /(1-$ a) resulting in a series of peaks. Unlike the feedforward case the curves are not symmetrical about the line $|H(\omega)|=b_{0}$; and the filter unstable near $a=1$.

A generalized comb filter (GCF) with both feedforward and feedback coefficients can effectively recognize protein coding region with pole radius $r=0.992$ (close to unity) and $L=3$ having Numerator coefficients $=\left[\begin{array}{lll}1 & 0 & -r^{L}\end{array}\right]$ and Denominator coefficients $=\left[\begin{array}{cccc}1 & 0 & 0 & -r^{L}\end{array}\right]$. The frequency response plot in Figure 4(a) shows a sharp peak at $\omega=2 \pi / 3$ which exhibit period-3 property.

\subsection{Design of an Improved Comb Filter for Prediction of Protein Coding Regions}

The CDC filter consists of equal number of stages of differentiators and comb filters in cascade. Hence we have referred to it is as cascaded differentiator-comb filter. It requires no multiplication and it can be designed with only adders, hence it can be preferred as a computationally efficient predictor for protein coding region. Since the CDC filter is followed by a down sampler for data rate down conversion, and can be called as CDC decimator filter.

\subsubsection{Single-Stage CDC Decimator}

The basic unit of a CDC decimator filter consists of a single stage of differentiator and a comb filter followed by a resampling switch as shown in Figure 5. The differentiator section operates at the high sampling rate $f$ and it is implemented as a one-zero filter with a unity feedforward coefficient. The difference equation and the corresponding transfer function, $H_{D}(z)$ for this section can be expressed in the form:

$$
\begin{gathered}
y(n)=x(n)-x(n-1) \\
Y(z)=X(z)-z^{-1} X(z) \\
H_{D}(z)=1-z^{-1}
\end{gathered}
$$

The comb section operates at the low sampling rate

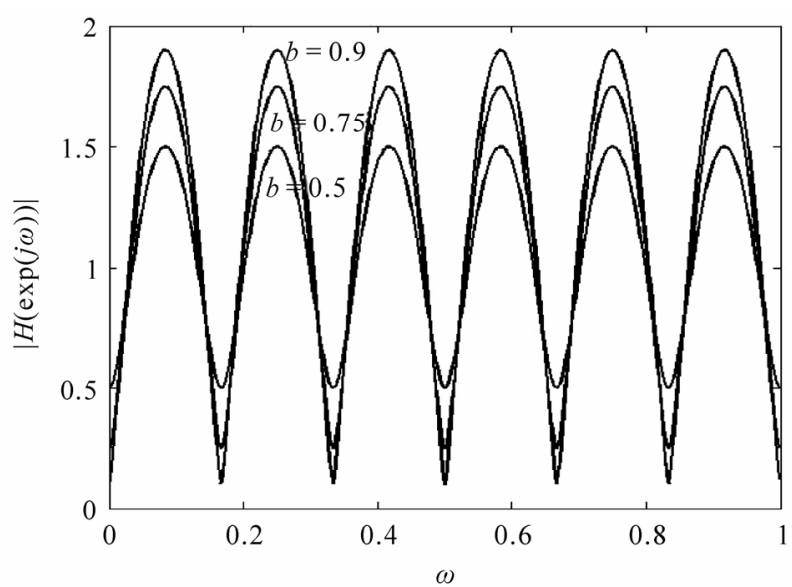

(a)

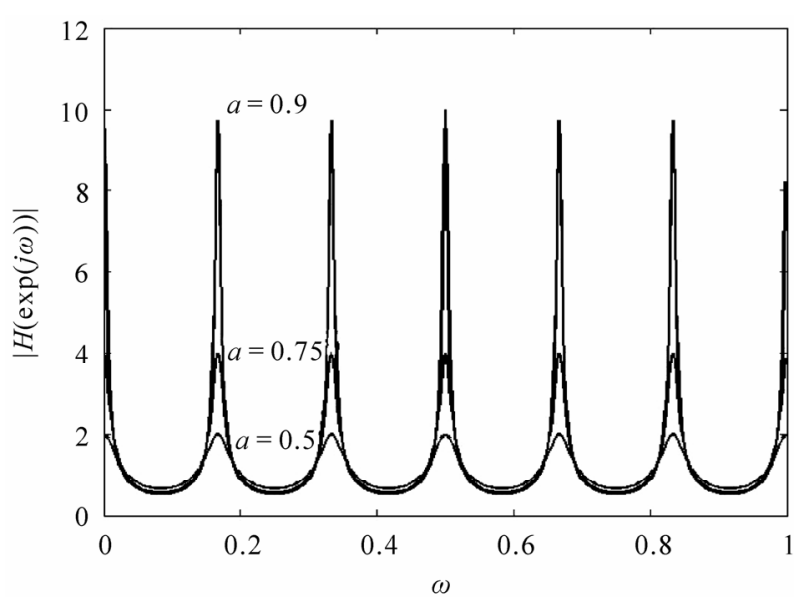

(b)

Figure 3. Magnitude response of comb filter (a) Feedforward (b) Feedback comb filter.

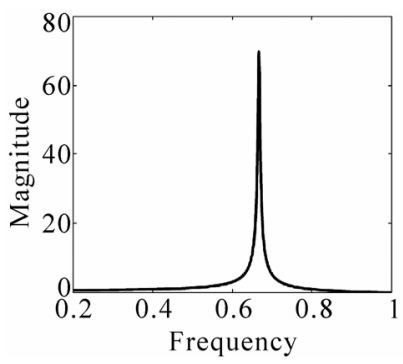

(a)

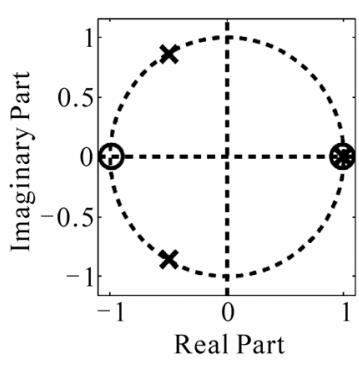

(b)
Figure 4. Characteristics of generalized comb filter. (a) Frequency response, (b) Pole-zero plot.

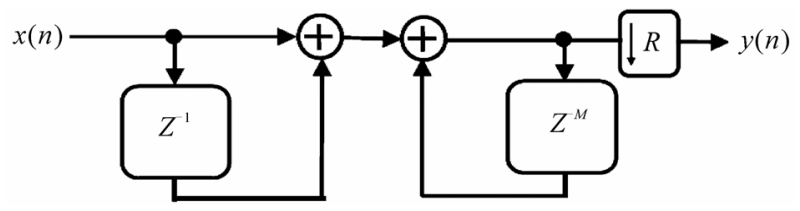

Figure 5. Single stage CDC Decimator. 
$f_{s} / R$ where $R$ is the integer rate change factor. This section consists of IIR comb stage with a delay of $M$ samples per stage with unity feedback coefficient. The differential delay is a filter design parameter used to control the filter's frequency response. The delay $M=3$ in the comb section is defined to exhibit period-3 property.

The difference equation and the corresponding transfer function, $H_{C}(z)$ for a single comb stage with a sample rate $R$ are given by

$$
\begin{gathered}
y(n)=x(n)+y(n-R M) \\
Y(z)=X(z)+z^{-R M} Y(z) \\
H_{c}(z)=\frac{1}{1-z^{-R M}}
\end{gathered}
$$

The decimator subsamples the output of the last stage, reducing the sample rate from $f_{s}$ to $f_{\mathrm{s}} / R$. The system transfer function for the composite CDC filter is given by

$$
H(z)=H_{D}(z) H_{C}(z)=\frac{1-z^{-1}}{1-z^{-R M}}
$$

\subsubsection{Frequency Response of CDC Filter}

The frequency response is obtained by evaluating Equation (18) at

$$
z=\mathrm{e}^{\frac{j 2 \pi f}{R}}
$$

where $f$ is the frequency relative to the sampling rate $f_{S} / R$. Evaluating Equation (18) in the $z$-plane at the sample points defined by Equation (19), gives the magnitude frequency response as

$$
|H(f)|=\frac{\left(\sin \frac{\pi f}{R}\right)}{\sin (\pi M f)}
$$

The frequency response plot and pole-zero plot of single stage CDC filter are shown in Figures 6(a) and 6(b) respectively. The frequency response plot shows a sharp peak at period-3 region. This property is employed for prediction of protein coding region.

\subsubsection{N-Stage CDC Filter}

A CDC decimator can in general be designed with $N$ cascaded differentiator stages clocked at $f_{s}$, followed by $N$ cascaded IIR comb stages running at $f_{s} / R$. Figure 7 shows three-stage CDC filter that consists of three numbers of differentiators and comb filters. Similarly block diagram of $N$-stage CDC Decimator Filter consisting of $N$ equal number of differentiators and IIR comb filter sections and the respective transfer functions are shown in Figures 8 and 9 respectively. The overall function of CDC filter with $N$ cascaded stages, (Figure 9) is given by

$$
H(z)=H_{D}^{N} H_{C}^{N}(z)=\left[\frac{1-z^{-1}}{1-z^{-R M}}\right]^{N}
$$

where $N$ is the number of differentiator-comb filter pairs. From (21), we observe that the CDC filter is equivalent to a cascade of $N$ uniform filter stages with unit coefficients. As part of the filter design process; $R, M$, and $N$ are chosen suitably to provide period-3 characteristic. For $M=3$ and $N=1$, the CDC exhibits period-3 behavior.

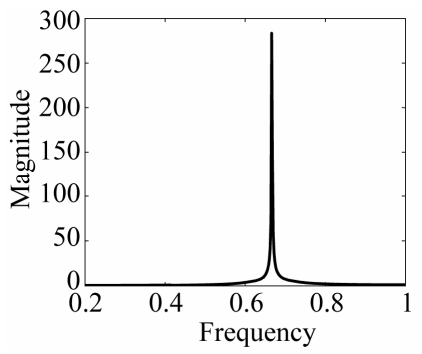

(a)

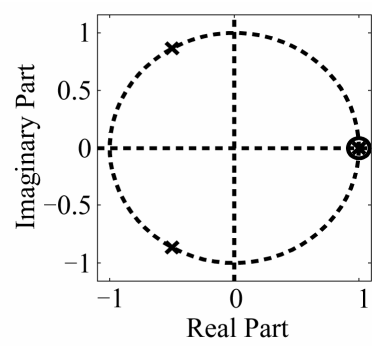

(b)
Figure 6. (a) Frequecy response (b) Pole-Zero plots of CDC filter.

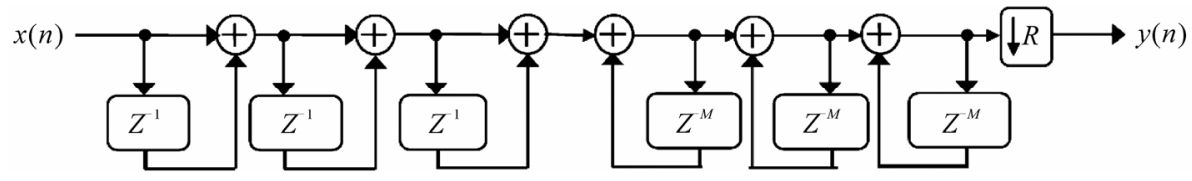

Figure 7. Block diagram of three stage CDC filter consisting of three equal number of differentiators and IIR comb filter stages followed by decimator.

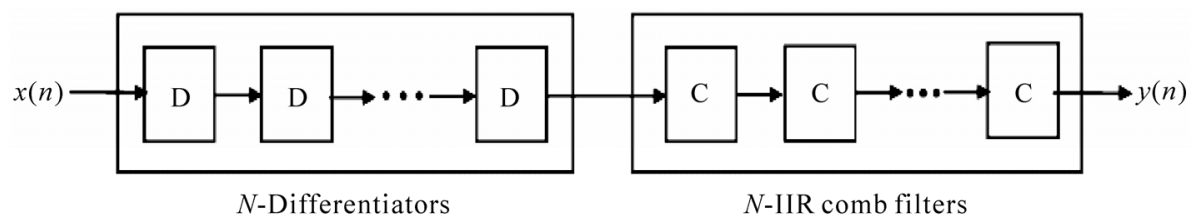

Figure 8. Block Diagram of $N$-stage CDC decimator filter consisting of $N$ equal number of differentiators and IIR comb filter sections. 


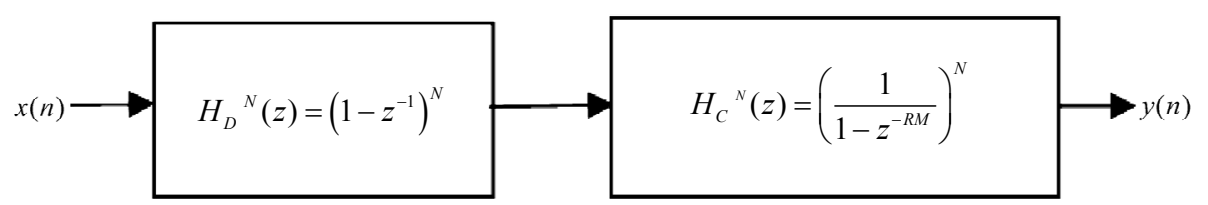

Figure 9. Block diagram of overall transfer function of $N$-stage differentiators and IIR comb filter sections.

The frequency response plot shows high peak at period-3 region. Thus single-stage CDC filter is sufficient for prediction of exons. With more number of stages, i.e, $N=2$ or 3 , the magnitude increases considerably and even exons having short sequences can be detected.

\section{Performance and Complexity Comparison}

The genomic sequences of several genes of different organisms were taken for the prediction of protein coding region using the proposed $\mathrm{GCF}$ and $\mathrm{CDC}$ filter and the existing signal processing techniques such as DFT, antinotch filter with frame size of 351 nucleotides. The single indicator sequence using paired numeric properties of nucleotides is used as numerical representation [13]. Mainly, three data sets are used as bench mark for this purpose such as the dataset prepared by Burset and Guigo [28], HMR195 prepared by Sanja Rogic [29] and KEGG gene sequence database prepared by M. Kanehisa and S. Goto [30]. In a good number of cases all the proposed methods performed well. The performance analysis of various methods can be made by prediction measures such as exon-intron discrimination factor $D$ [10], sensitivity $\left(S_{N}\right)$, specificity $\left(S_{P}\right)$, miss rate $\left(M_{R}\right)$ and wrong rate $\left(W_{R}\right)[1,25]$ which are defined as follows:

$$
\begin{array}{r}
D=\frac{\text { Lowest of exon pe }}{\text { Highest peak in noncoding }} \\
S_{P}=\frac{T_{P}}{T_{P}+F_{P}} \\
S_{N}=\frac{T_{P}}{T_{P}+F_{N}} \\
M_{R}=\frac{M E}{A E} \\
W_{R}=\frac{W E}{P E}
\end{array}
$$

where $M E=$ missing exons, $A E=$ actural exons, $W E=$ wrong exons, $P E=$ predicted exons, $T_{P}=$ true positive, $F_{P}=$ false positive and $F_{N}=$ false negative [31]. $T_{P}$ corresponds to those genes that are correctly predicted by the algorithm and also exist in the GenBank annotation. $F_{P}$ corresponds to the coding regions identified by a given algorithm which are not present in the standard anno- tation. $F_{N}$ is coding region that is present in the GenBank annotation but not predicted to be coding by the algorithm being used. Higher the value of $D$ better is the discrimination. If $D$ is more than one $(D>1)$, all exons are identified without ambiguity. High sensitivity and specificity are desirable for higher accuracy.

The list of genes under study of different datasets and the performance analysis of various DSP approaches are shown in respective Tables. Table 1 summarizes the simulation results of nine genes from Burset and Guigo dataset whereas Table 2 summarizes the observations of six genes from KEGG dataset. Table $\mathbf{3}$ summarizes the observations of nine genes from HMR195 dataset. In all the examples cited the proposed encoding methods show better discrimination compared to the exising methods. The simulation result shows high discriminating factor, sensitivity and specificity with low miss rate and wrong rate for the proposed methods.

The proposed GCF and CDC filtering show high peak at exon locations in compared to existing methods as shown in figures. Figure 10 shows the exon prediction results for gene F56F11.4a with accession no: AF099922 in the C. elegans chromosome-III. Figures 10(a)-(c) show, respectively, the response of DFT, allpass-based antinotch filter with pole radius $r=0.992$ and the generalized comb filter with both feedforward and feedback coefficients having pole radius $r=0.992$ respectively. The five peaks corresponding to the exons can be seen at the respective locations $(1 \cdots 111,1600 \cdots 1929,3186 \cdots$ $3449,4537 \cdots 4716,6329 \cdots 6677)$. Figures 11(a)-(c) show the response of basic CDC filter of one stage, two stages and three stages CDC filter respectively. Figure 11 shows the response of CDC filter which has detected all the exons effectively and also shows higher magnitudes as compared to other signal processing methods. It is found that even one stage CDC filter produces comparatively higher magnitude than other methods at the respective exon locations. Hence one stage CDC filter is sufficient to act as efficient predictor which costs only two adders. As the number of cascaded stage increases, the magnitude of the frequency response plot also increases considerably. Figure 12 shows exon prediction result for gene PP32R1 with accession no: AF008216 of Homo sapiens consisting of one exon using DFT, allpass based antinotch filter and GCF methods. This indicates a 
Table 1. Prediction measures of DSP tools using burset and guigo dataset.

\begin{tabular}{|c|c|c|c|c|c|c|}
\hline \multirow{2}{*}{ Gene Name and Accession No } & \multirow{2}{*}{ DSP Methods } & \multicolumn{5}{|c|}{ Prediction Measures } \\
\hline & & $D$ & $S_{N}$ & $S_{P}$ & $M_{R}$ & $W_{R}$ \\
\hline \multirow{4}{*}{ PP32R1, AF00A216, Homo Sapiens } & DFT & 7.5 & 1 & 0.5 & 0 & 0.5 \\
\hline & Antinotch Filter & 7 & 1 & 1 & 0 & 0 \\
\hline & $\mathrm{GCF}$ & 12 & 1 & 1 & 0 & 0 \\
\hline & CDC Filter & 18 & 1 & 1 & 0 & 0 \\
\hline \multirow{4}{*}{$\begin{array}{l}\text { ALOEGLOBIN L25370, Alouatta } \\
\text { belzebul epsilon-globin gene }\end{array}$} & DFT & 1 & 1 & 0.5 & 0 & 0.5 \\
\hline & Antinotch Filter & 1.5 & 1 & 0.66 & 0 & 0.5 \\
\hline & GCF & 2.2 & 1 & 1 & 0 & 0 \\
\hline & CDC Filter & 3.5 & 1 & 1 & 0 & 0 \\
\hline \multirow{4}{*}{ Humbetgloa, 26462, human betaglobin } & DFT & 1.1 & 1 & 0.6 & 0 & 0.66 \\
\hline & Antinotch Filter & 1.2 & 1 & 0.75 & 0 & 0.33 \\
\hline & GCF & 1.25 & 1 & 0.75 & 0 & 0.33 \\
\hline & CDC Filter & 1.5 & 1 & 0.75 & 0 & 0.33 \\
\hline \multirow{4}{*}{$\begin{array}{l}\text { AGU04852 U04852 Ateles geoffroyi } \\
\text { haptoglobin (Hp) gene }\end{array}$} & DFT & 1 & 1 & 0.6 & 0 & 0.66 \\
\hline & Antinotch Filter & 1.2 & 1 & 0.75 & 0 & 0.33 \\
\hline & GCF & 1.45 & 1 & 0.75 & 0 & 0 \\
\hline & CDC Filter & 2.5 & 1 & 1 & 0 & 0 \\
\hline \multirow{4}{*}{ Humelafin, D13156, Homo Sapiens } & DFT & 0.71 & 1 & 0.5 & 0 & 0.5 \\
\hline & Antinotch Filter & 2.5 & 1 & 1 & 0 & 0 \\
\hline & GCF & 2.91 & 1 & 1 & 0 & 0 \\
\hline & CDC Filter & 3.2 & 1 & 1 & 0 & 0 \\
\hline \multirow{4}{*}{$\begin{array}{l}\text { G101 U12024 Astyanax mexicanus } \\
\text { green opsin gene }\end{array}$} & DFT & 1.1 & 1 & 0.66 & 0 & 0.5 \\
\hline & Antinotch Filter & 2.3 & 1 & 1 & 0 & 0 \\
\hline & GCF & 2.35 & 1 & 1 & 0 & 0 \\
\hline & CDC Filter & 2.75 & 1 & 1 & 0 & 0 \\
\hline \multirow{4}{*}{$\begin{array}{l}\text { HUMCBRG, M62420, carbonyl } \\
\text { reductase gene }\end{array}$} & DFT & 0.8 & 0.6 & 0.6 & 0.33 & 0.33 \\
\hline & Antinotch Filter & 0.8 & 0.6 & 0.6 & 0.33 & 0.33 \\
\hline & GCF & 1 & 1 & 1 & 0 & 0 \\
\hline & CDC Filter & 1 & 1 & 1 & 0 & 0 \\
\hline \multirow{4}{*}{$\begin{array}{l}\text { BOVANPA M13145 Bovine atrial } \\
\text { natriuretic peptide }\end{array}$} & DFT & 1.2 & 1 & 1 & 0 & 0.66 \\
\hline & Antinotch Filter & 1.2 & 1 & 1 & 0 & 0.66 \\
\hline & GCF & 1.5 & 1 & 1 & 0 & 0.33 \\
\hline & CDC Filter & 2.5 & 1 & 1 & 0 & 0.33 \\
\hline \multirow{4}{*}{ HSABLGR1 U07561 Human ABL gene } & DFT & 0.8 & 0.5 & 0.5 & 0 & 0.5 \\
\hline & Antinotch Filter & 1.1 & 1 & 0.7 & 0 & 0.42 \\
\hline & GCF & 2.1 & 1 & 0.87 & 0 & 0.14 \\
\hline & CDC Filter & 2.5 & 1 & 1 & 0 & 0 \\
\hline
\end{tabular}


Table 2. Prediction measures of DSP tools using KEGG dataset.

\begin{tabular}{|c|c|c|c|c|c|c|}
\hline \multirow{2}{*}{ Gene Name and Accession No } & \multirow{2}{*}{ DSP Methods } & \multicolumn{5}{|c|}{ Prediction Measures } \\
\hline & & $D$ & $S_{P}$ & $S_{N}$ & $M_{R}$ & $W_{R}$ \\
\hline \multirow{4}{*}{$\begin{array}{l}\text { NC_004843 Buchnera aphidicola } \\
\text { Ps plasmid pBPS1 }\end{array}$} & DFT & 0.6 & 0.5 & 1 & 0 & 0.5 \\
\hline & Antinotch Filter & 0.5 & 0.5 & 1 & 0 & 0.5 \\
\hline & GCF & 1 & 0.5 & 1 & 0 & 0.33 \\
\hline & CDC Filter & 1.1 & 0.66 & 1 & 0 & 0.33 \\
\hline \multirow{4}{*}{$\begin{array}{l}\text { NC_001911 Buchnera aphidicola } \\
\text { Dn plasmid pLeu-Dn }\end{array}$} & DFT & 1.1 & 0.63 & 1 & 0 & 0.4 \\
\hline & Antinotch Filter & 1.2 & 0.63 & 1 & 0 & 0.4 \\
\hline & GCF & 1.2 & 0.7 & 1 & 0 & 0.33 \\
\hline & CDC Filter & 1.5 & 0.7 & 1 & 0 & 0.28 \\
\hline \multirow{4}{*}{$\begin{array}{l}\text { NC_002650 Treponema denticola U9b } \\
\text { plasmid pTS1 }\end{array}$} & DFT & 1.2 & 0.6 & 1 & 0 & 0.33 \\
\hline & Antinotch Filter & 1.5 & 0.6 & 1 & 0 & 0.33 \\
\hline & GCF & 3 & 1 & 1 & 0 & 0 \\
\hline & CDC Filter & 3.5 & 1 & 1 & 0 & 0 \\
\hline \multirow{4}{*}{$\begin{array}{l}\text { NC_007142 Campylobacter coli } 338 \\
\text { plasmid p3384 }\end{array}$} & DFT & 1.15 & 0.6 & 1 & 0 & 0.5 \\
\hline & Antinotch Filter & 1.3 & 0.6 & 1 & 0 & 0.5 \\
\hline & GCF & 1.32 & 0.75 & 1 & 0 & 0.33 \\
\hline & CDC Filter & 1.4 & 0.75 & 1 & 0 & 0.33 \\
\hline \multirow{4}{*}{$\begin{array}{l}\text { NC_004767 Helicobacter pylori } \\
\text { plasmid pHP51 }\end{array}$} & DFT & 1.2 & 1 & 0.5 & 0.5 & 0 \\
\hline & Antinotch Filter & 1.2 & 0.5 & 1 & 0 & 0 \\
\hline & GCF & 1.2 & 0.5 & 1 & 0 & 0 \\
\hline & CDC Filter & 1.3 & 1 & 1 & 0 & 0 \\
\hline \multirow{4}{*}{$\begin{array}{l}\text { NC_010099 Burkholderia cepacia } \\
\text { plasmid PPC1 }\end{array}$} & DFT & 0.8 & 0.33 & 1 & 0 & 0.66 \\
\hline & Antinotch Filter & 3 & 0.5 & 1 & 0 & 0.5 \\
\hline & GCF & 4.1 & 1 & 1 & 0 & 0 \\
\hline & CDC Filter & 6.5 & 1 & 1 & 0 & 0 \\
\hline
\end{tabular}

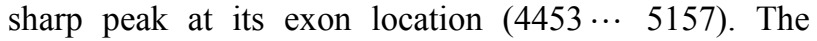
same gene has been injected to CDC filters of different stages. The corresponding responses are shown in Figure 13.

The generalized comb filter and CDC filter sense the exons effectively by showing high peak at gene locations with lower computation. Table 4 summarizes the comparison of computational complexities of the proposed comb filter based gene prediction method with existing approaches. The generalized comb filter and the CDC filter have lower computational complexity. Again these methods detect all the exons at their respective locations. CDC filter based technique is found to be more efficient than other approaches for gene prediction in terms of prediction efficiency as well as the computational complexity.

\section{Conclusions}

The proposed novel comb filter-based approaches for the prediction of protein coding regions of DNA sequences using the period-3 property have better prediction efficiency and lower computational complexity. The GCF as well as the CDC filters are found to detect the exons with considerably sharp peak at protein coding regions for eukaryotic cell and can predict the specific exons with high discriminating factor, sensitivity and specificity and low miss rate and wrong rate. The CDC approach can detect smaller exon regions having short sequences. It 
Table 3. Prediction measures of DSP tools using HMR195 dataset.

\begin{tabular}{|c|c|c|c|c|c|c|}
\hline \multirow{2}{*}{ Gene Name and Accession No } & \multirow{2}{*}{ DSP Methods } & \multicolumn{5}{|c|}{ Prediction Measures } \\
\hline & & $D$ & $S_{N}$ & $S_{P}$ & $M_{R}$ & $W_{R}$ \\
\hline \multirow{4}{*}{$\begin{array}{l}\text { FABP3 U17081 Human fatty acid } \\
\text { binding protein }\end{array}$} & DFT & 1.1 & 1 & 0.66 & 0 & 0.5 \\
\hline & Antinotch Filter & 2.2 & 1 & 1 & 0 & 0.5 \\
\hline & GCF & 3.05 & 1 & 1 & 0 & 0 \\
\hline & CDC Filter & 3.25 & 1 & 1 & 0 & 0 \\
\hline \multirow{4}{*}{$\begin{array}{l}\text { SIX3, AF092047, Homo Sapiens } \\
\text { Homeobox protein }\end{array}$} & DFT & 1 & 1 & 0.66 & 0 & 0.5 \\
\hline & Antinotch Filter & 1 & 1 & 0.66 & 0 & 0.5 \\
\hline & GCF & 1.02 & 1 & 0.66 & 0 & 0.5 \\
\hline & CDC Filter & 1.25 & 1 & 0.66 & 0 & 0.5 \\
\hline \multirow{4}{*}{$\begin{array}{l}\text { Osteomodulin AB009589 Human gene } \\
\text { for Osteomodulin }\end{array}$} & DFT & 1.2 & 1 & 0.66 & 0 & 0.5 \\
\hline & Antinotch Filter & 2 & 1 & 1 & 0 & 0 \\
\hline & GCF & 2.25 & 1 & 1 & 0 & 0 \\
\hline & CDC Filter & 2.85 & 1 & 1 & 0 & 0 \\
\hline \multirow{4}{*}{ KIP AB021866 Homo sapiens KIP gene } & DFT & 1 & 1 & 0.5 & 0 & 0.5 \\
\hline & Antinotch Filter & 1.2 & 1 & 0.5 & 0 & 0.5 \\
\hline & GCF & 2 & 1 & 0.5 & 0 & 0.5 \\
\hline & CDC Filter & 2.25 & 1 & 0.5 & 0 & 0.5 \\
\hline \multirow{4}{*}{$\begin{array}{l}\text { CLDN3, AF007189, Homo sapiens } \\
\text { Claudin } 3\end{array}$} & DFT & 1.8 & 1 & 0.66 & 0 & 0.5 \\
\hline & Antinotch Filter & 2 & 1 & 1 & 0 & 0 \\
\hline & GCF & 2 & 1 & 1 & 0 & 0 \\
\hline & CDC Filter & 2.25 & 1 & 1 & 0 & 0 \\
\hline \multirow{4}{*}{$\begin{array}{l}\text { mafG, AB009693, Mus musculus gene } \\
\text { for mafG }\end{array}$} & DFT & 0.8 & 0.5 & 0.5 & 0.5 & 0 \\
\hline & Antinotch Filter & 1.25 & 1 & 1 & 0 & 0 \\
\hline & GCF & 2 & 1 & 1 & 0 & 0 \\
\hline & CDC Filter & 2.5 & 1 & 1 & 0 & 0 \\
\hline \multirow{4}{*}{$\begin{array}{l}\text { GalR2, AF042784, Musculus galin } \\
\text { receptor type } 2 \text { gene }\end{array}$} & DFT & 1 & 1 & 0.5 & 0 & 0.5 \\
\hline & Antinotch Filter & 1.2 & 1 & 0.5 & 0 & 0.5 \\
\hline & GCF & 1.2 & 1 & 0.5 & 0 & 0.5 \\
\hline & CDC Filter & 1.25 & 1 & 0.5 & 0 & 0.5 \\
\hline \multirow{4}{*}{$\begin{array}{l}\text { D p19, AFO61327, Homo sapiens } \\
\text { cyclin-dependent kinase4 inhibitor }\end{array}$} & DFT & 1.5 & 1 & 1 & 0 & 0.5 \\
\hline & Antinotch Filter & 2.6 & 1 & 1 & 0 & 0 \\
\hline & GCF & 3.75 & 1 & 1 & 0 & 0 \\
\hline & CDC Filter & 5.15 & 1 & 1 & 0 & 0 \\
\hline \multirow{4}{*}{$\begin{array}{l}\text { AF064081 Mus musculus } \\
\text { alpha-sarcoglycan gene }\end{array}$} & DFT & 0.75 & 0.5 & 0.5 & 0 & 0.37 \\
\hline & Antinotch Filter & 1.5 & 1 & 0.72 & 0 & 0.37 \\
\hline & GCF & 2 & 1 & 0.88 & 0 & 0.12 \\
\hline & CDC Filter & 2.5 & 1 & 1 & 0 & 0 \\
\hline
\end{tabular}




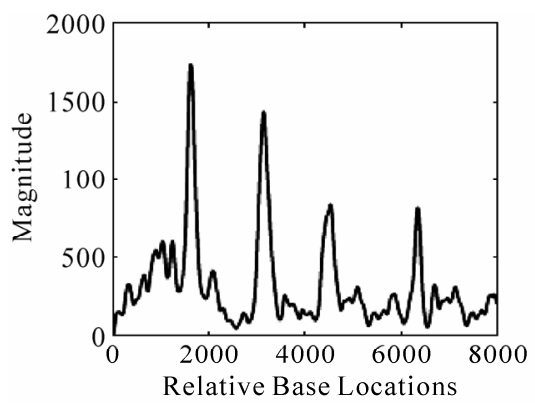

(a)

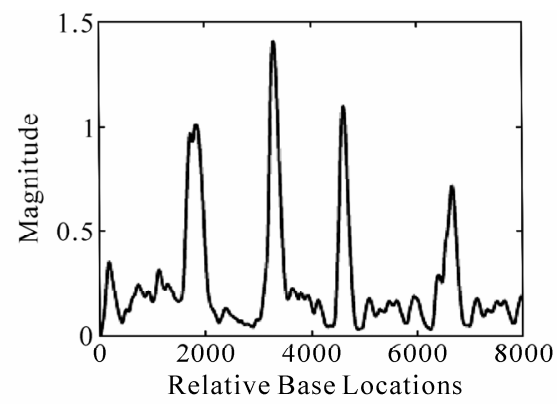

(b)

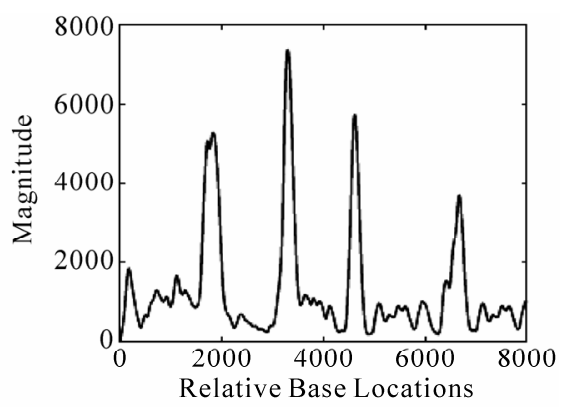

(c)

Figure 10. Gene F56F11.4a of C.Elegans chromosome III showing 5 exons by (a) DFT, (b) Antinotch filter, (c) Generalised Comb Filter (GCF).

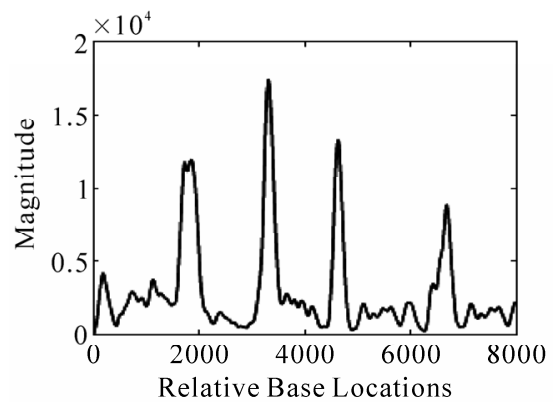

(a)

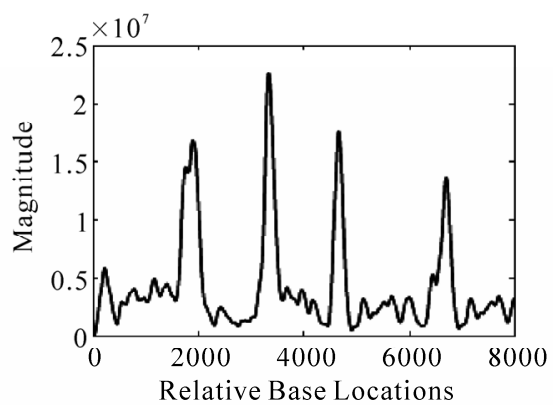

(b)

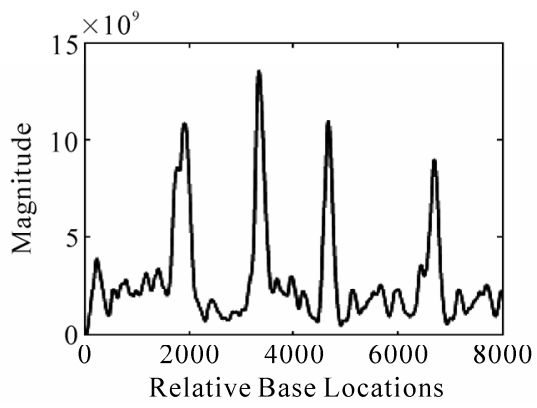

(c)

Figure 11. Gene F56F11.4a showing five exons by CDC filter with (a) Single stage $(N=1)(\mathrm{b})$ Two stages $(N=2)(\mathrm{c})$ Three stages $(N=3)$.

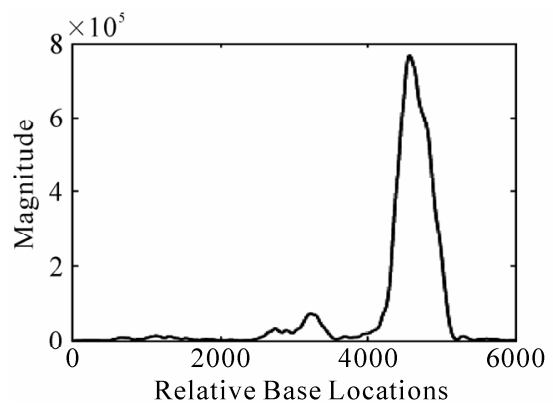

(a)

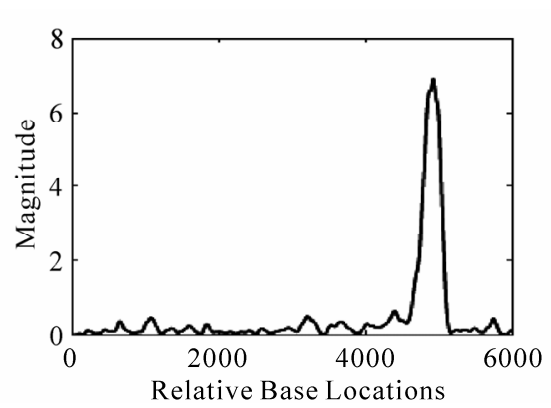

(b)

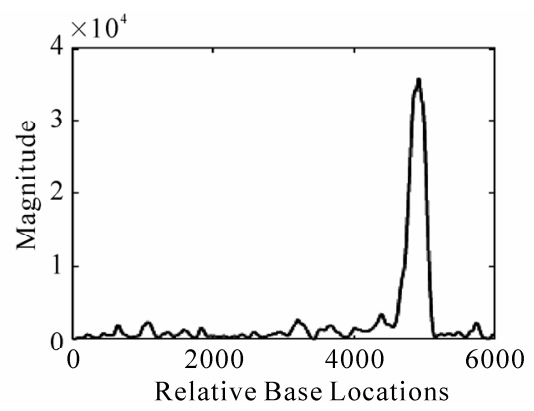

(c)

Figure 12. Gene PP32R1 of Homo sapiens showing one Exon by (a) DFT, (b) Antinotch filter, (c) Generalised Comb Filter.

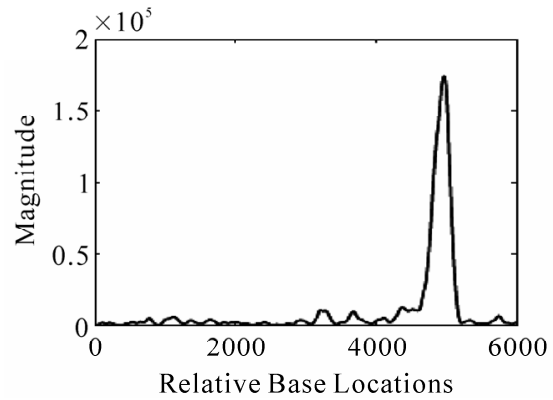

(a)

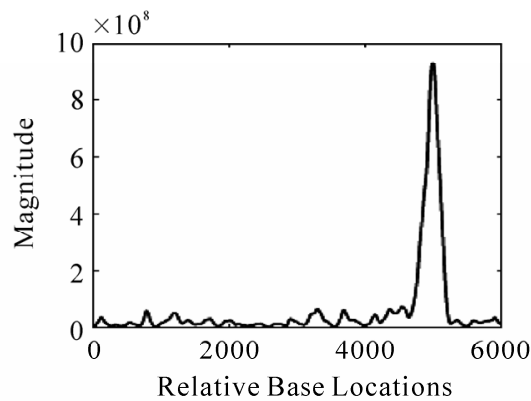

(b)

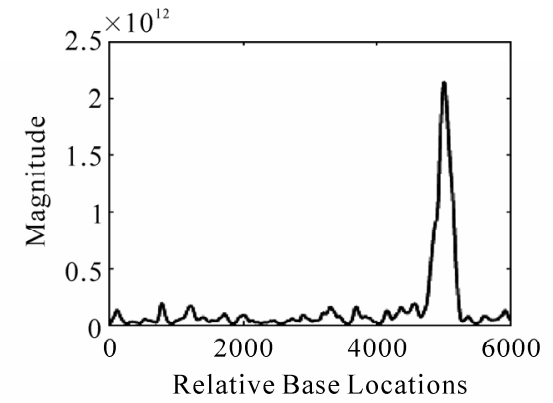

(c)

Figure 13. Gene PP32R1 of Homo sapiens showing one exon by CDC filter with (a) Single stage $(N=1)(b)$ Two stages $(N=2)$ (c) Three stages $(N=3)$. 
Table 4. Computational complexities of DSP methods for prediction of protein coding region using period-3 property.

\begin{tabular}{lcc}
\hline \multicolumn{1}{c}{ Prediction Technique } & Multiplications & Additions \\
\hline Using $N$-point DFT & $N^{2}(\mathrm{C})$ & $N(N-1)(\mathrm{C})$ \\
Using $N$-point FFT & $(N / 2) \log _{2} N(\mathrm{C})$ & $N \log _{2} N(\mathrm{C})$ \\
Allpass Antinotch Filtering & $(2 N+1)(\mathrm{R})$ & $2 N(\mathrm{R})$ \\
Multistage Filtering & $5(\mathrm{R})$ & $2 N(\mathrm{R})$ \\
Generalized Comb Filtering & $3(\mathrm{R})$ & $2(\mathrm{R})$ \\
CDC Filtering & $\mathrm{NIL}$ & $2(\mathrm{R})$ \\
\hline
\end{tabular}

'C' and ' $\mathrm{R}$ ' refer to complex and real arithmetic operations respectively.

can therefore be used as an efficient tool for the identification of protein coding regions of DNA sequences. As such comb filter is simple in structure, involves less computational complexity and better prediction efficiency compared to the FFT-based methods and other digital filtering methods. Hence it can be used as a computationally efficient and better alternative to other DSP approach to the prediction of protein coding regions.

\section{REFERENCES}

[1] Z. Wang, Y. Z. Chen and Y. X. Li, "A Brief Review of Computational Gene Prediction Methods," Genomics Proteomics Bioinformatics, Vol. 2, No. 4, 2004, pp. 216-221.

[2] D. Anastassiou, "Genomic Signal Processing," Signal Processing Magazine, Vol. 18, No. 4, 2001, pp. 8-20. doi:10.1109/79.939833

[3] J. W. Fickett, "The Gene Identification Problem: Overview for Developers," Computers \& Chemistry, Vol. 20, No. 1, 1996, pp. 103-118. doi:10.1016/S0097-8485(96)80012-X

[4] R. Voss, "Evolution of Long-Range Fractal Correlations and $1 / f$ Noise in DNA Base Sequences," Physical Review Letters, Vol. 68, No. 25, 1992, pp. 3805-3808. doi:10.1103/PhysRevLett.68.3805

[5] P. D. Cristea, "Genetic signal Representation and Analysis," Proceedings of SPIE Conference, International Biomedical Optics Symposium (BIOS'02), Vol. 4623, 2002, pp. 77-84.

[6] A. K. Brodzik and O. Peters, "Symbol-Balanced Quaternionic Periodicity Transform for Latent Pattern Detection in DNA Sequences," IEEE International Conference on Acoustics, Speech, and Signal Processing (ICASSP '05), Vol. 5, 2005, pp. 373-376.

[7] T. M. Nair, S. S. Tambe and B. D. Kulkarni, "Application of Artificial Neural Networks for Prokaryotic Transcription Terminator Prediction," FEBS Letters, Vol. 346, No. 2-3, 1994, pp. 273-277. doi:10.1016/0014-5793(94)00489-7

[8] A. S. Nair and S. P. Sreenathan, "A Coding Measure
Scheme Employing Electron-Ion Interaction Pseudopotential (EIIP)," Bioinformation, Vol. 1, No. 6, 2006, pp. 197-202.

[9] G. L. Rosen, "Signal Processing for Biologically-Inspired Gradient Source Localization and DNA Sequence Analysis," Ph.D. Thesis, Georgia Institute of Technology, Atlanta, 2006.

[10] A. S. Nair and S. P. Sreenathan, "An Improved Digital Filtering Technique Using Frequency Indicators for Locating Exons," Journal of the Computer Society of India, Vol. 36, No. 1, 2006.

[11] R. Zhang and C. T. Zhang, "Z Curves, an Intuitive Tool for Visualizing and Analyzing the DNA Sequences," Journal of Biomolecular Structure \& Dynamics, Vol. 11, No. 4, 1994, pp. 767-782.

[12] A. Rushdi and J. Tuqan, "Gene Identification Using the Z-Curve Representation," IEEE International Conference on Acoustics, Speech and Signal Processing, Toulouse, 14-19 May 2006, pp. 1024-1027.

[13] M. Akhtar, J. Epps and E. Ambikairajah, "On DNA Numerical Representations for Period-3 Based Exon Prediction," IEEE International Workshop on Genomic Signal Processing and Statistics, Tuusula, 2007.

[14] B. D. Silverman and R. Linsker, "A Measure of DNA Periodicity," Journal of Theoretical Biology, Vol. 118, No. 3, 1986, pp. 295-300. doi:10.1016/S0022-5193(86)80060-1

[15] S. Tiwari, S. Ramachandran, A. Bhattacharya, S. Bhattacharya and R. Ramaswamy, "Prediction of Probable Genes by Fourier Analysis of Genomic Sequences," Bioinformatics, Vol. 13, No. 3, 1997, pp. 263-270. doi:10.1093/bioinformatics/13.3.263

[16] D. Anastassiou, "Digital Signal Processing of Biomolecular Sequences," Technical Report, Columbia University, 2000-20-041, April 2000.

[17] D. Anastassiou, "Frequency-Domain Analysis of Biomolecular Sequences," Bioinformatics, Vol. 16, No. 12, 2000, pp. 1073-1082.

doi:10.1093/bioinformatics/16.12.1073

[18] P. P. Vaidyanathan and B. J. Yoon, "Digital Filters for Gene Prediction Applications," IEEE Asilomar on Signals, Systems, and Computers, Monterey, 3-6 November 2002, pp. 306-310.

[19] P. P. Vaidyanathan and B. J. Yoon, "The Role of Signal Processing Concepts in Genomics and Proteomics," Journal of the Franklin Institute, Vol. 341, No. 1-2, 2004, pp. 111-135. doi:10.1016/j.jfranklin.2003.12.001

[20] D. Koltar and Y. Lavner, "Gene Prediction by Spectral Rotation (SR) Measure: A New Method for Identifying Protein-Coding Regions," Genome Research, Vol. 13, No. 8, 2003, pp. 1930-1937.

[21] A. Fuentes, J. Ginori and R. Abalo, "A New Predictor of Coding Regions in Genomic Sequences Using a Combination of Different Approaches," International Journal of Biomedical and Life Sciences, Vol. 3, No. 2, 2007, pp. $1-5$. 
[22] J. Tuqan and A. Rushdi, "A DSP Approach for Finding the Codon Bias in DNA Sequences," IEEE Journal of Selected Topics in Signal Processing, Vol. 2, No. 3, 2008, pp. 343-356. doi:10.1109/JSTSP.2008.923851

[23] P. Jesus, M. Chalco and H. Carrer, "Identification of Protein Coding Regions Using the Modified Gabor-Wavelet Tranform," IEEE/ACM Transaction on Computational Biology and Bioinformatics, Vol. 5, No. 2, 2008, pp. 198207. doi:10.1109/TCBB.2007.70259

[24] L. Galleani and R. Garello, "The Minimum Entropy Mapping Spectrum of a DNA Sequence," IEEE Transaction on Information Theory, Vol. 56, No. 2, 2010, pp. 771-783. doi:10.1109/TIT.2009.2037041

[25] M. Akhtar, J. Epps and E. Ambikairajah, "Signal Processing in Sequence Analysis: Advances in Eukaryotic Gene Prediction," IEEE Journal of Selected Topics in Signal Processing, Vol. 2, No. 3, 2008, pp. 310-321. doi:10.1109/JSTSP.2008.923854

[26] S. K. Mitra, "Digital Signal Processing," Tata McGraw-Hill, New Delhi, 2006.
[27] A. V. Oppenheim and R. W. Schafer, "Discrete-Time Signal Processing," Prentice-Hall Inc., Upper Saddle River, 1999.

[28] M. Burset and A. R. Guigo, "Evaluation of Gene Structure Prediction Programs," Genomics, Vol. 34, No. 3, 1996, pp. 353-367. doi:10.1006/geno.1996.0298

[29] S. Rogic, A. Mackworth and F. Ouellette, "Evaluation of Gene Finding Programs on Mammalian Sequences," Genome Research, Vol. 11, No. 5, 2001, 817-832. doi:10.1101/gr.147901

[30] M. Kanehisa and S. Goto, "KEGG: Kyoto Encyclopedia of Genes and Genomes," Nucleic Acid Research, Vol. 28, No. 1, 2000, pp. 27-30. doi:10.1093/nar/28.1.27

[31] G. Aggarwal and R. Ramaswamy, “Ab Initio Gene Identification: Prokaryote Genome Annotation with GeneScan and GLIMMER," Journal of Biosciences, Vol. 27, No. 1, 2002, pp. 7-14. doi:10.1007/BF02703679 